\title{
A receptor-like kinase enhances sunflower resistance to Orobanche cumana
}

Pauline Duriez ${ }^{1,2}$, Sonia Vautrin ${ }^{3}$, Marie-Christine Auriac ${ }^{1}$, Julia Bazerque ${ }^{1}$, Marie-Claude Boniface ${ }^{1}$, Caroline Callot $^{3}$, Sébastien Carrère ${ }^{1}$, Stéphane Cauet ${ }^{3}$, Mireille Chabaud ${ }^{1}$, Fabienne Gentou ${ }^{2}$, Marta Lopez-Sendon², Clémence Paris ${ }^{2}$, Prune Pégot-Espagnet ${ }^{1}$, Jean-Christophe Rousseaux², Begoña PérezVich $^{4}$, Leonardo Velasco ${ }^{4}$, Hélène Bergès ${ }^{3}$, Joël Piquemal ${ }^{2}$, Stéphane Muños ${ }^{1 *}$.

1. LIPM, Université de Toulouse, INRA, CNRS, Castanet-Tolosan, France

2. Syngenta Seeds, Saint-Sauveur, France

3. CNRGV, INRA, Castanet-Tolosan, France

4. IAS-CSIC, Córdoba, Spain

*corresponding author: stephane.munos@inra.fr

\begin{abstract}
:
Orobanche cumana (sunflower broomrape) is an obligate parasitic plant that infects sunflower roots, causing yield losses. Using a map-based cloning strategy, we identified the HaOr7 resistance gene to O. cumana race $F$, which was found to encode a LRR receptor-like kinase. The complete HAOR7 protein was present in resistant lines and prevented $O$. cumana from connecting to the vascular system of sunflower roots, while susceptible lines encoded a truncated protein lacking transmembrane and kinase domains.
\end{abstract}


Plants are known to interact with each other, but the genetic and the molecular mechanisms of plantplant interactions are still poorly described ${ }^{1}$. In the extreme case, parasitic plants depend on other plants to complete their life cycle. There are more than 3,000 parasitic plant species, including hemiparasites that are only partly parasitic, and holoparasites ${ }^{2}$ that are completely dependent on their host for carbon. Parasitic plants attach to either the stems or the roots of the host, thus connecting to the host plant through a haustorium that enables penetration in the host plant tissues ${ }^{3}$.

Among the most studied parasitic plants are the Cuscuta (dodder), Striga (witchweeds) and Orobanche (broomrapes) genera, because some species in each group parasitize cultivated plants of economic importance. Depending on the level of field infestation, crop yield losses can be severe ${ }^{4}$. Parasitic plant species vary in degree of host specificity, and sunflower is the only crop parasitized by Orobanche cumana. While some of the genetic and/or molecular determinants of resistance have been described for Striga ${ }^{5}$ and Orobanche ${ }^{6}$, no resistance genes to $O$. cumana have been identified to date. However, a number of genetic mapping studies have analyzed quantitative resistance to $O$. cumana in sunflower, and several QTLs have been shown to be specific to one or more developmental stages ${ }^{7}$ or to control the number of broomrape plants that emerge ${ }^{8}$.

There are also major resistance genes named Or that have been used for decades in breeding programs ${ }^{9}$. However, only the $\operatorname{Or} 5$ gene has previously been mapped. The Or5 gene is located on LG3 ${ }^{10}$ and has been shown to interact with the AvrOr5 avirulence gene ${ }^{11}$, consistent with the gene-for-gene model $^{12}$. Broomrape populations continue to evolve and overcome the resistance conferred by $\mathrm{Or}$ genes that have been introduced into commercial sunflower varieties, as was recently reported in Spain $^{13}$. This rapid evolution has increased the level of virulence as indicated by resistance profiles of a set of differential sunflower lines ${ }^{14}$. Since the early 2000 's, several commercial sunflower hybrids were fully resistant to $O$. cumana race $\mathrm{F}$ by the introgression of the $\operatorname{Or} 7$ gene, named $\mathrm{HaOr} 7$ in our study.

Here, we describe the cloning of the HaOr7 gene that controls resistance to 0 . cumana in sunflowers by combining large-scale genetic and genomic approaches. We report that HaOr7 encodes a leucinerich repeat receptor-like kinase protein that is truncated in susceptible sunflowers. The fully functional HAOR7 protein originates from a wild Helianthus accession and confers resistance during the early stages of the interaction. 
For the mapping of the HaOr7 gene onto the sunflower genome, we selected a panel of 55 cultivated sunflower lines. This panel consisted of unrelated lines and was divided into 21 susceptible HaOr7- and 34 resistant HaOr7+ lines into which the HaOr7 gene resistance allele had been introgressed from the same original source. We phenotyped these lines in the field and genotyped them using an $\mathrm{AXIOM}^{\circledR}$ chip. Using both genotyping (305,201 SNPs) and phenotyping data, GWA analysis revealed a unique locus located on chromosome 7 (Fig. 1a) in a genomic region of 1,122,175 bp (https://www.heliagene.org/HanXRQ-SUNRISE/) ${ }^{15}$. We confirmed the mapping of the HaOr7 gene to this region of chromosome 7 using a population of 355 recombinant inbred lines (Fig. 1b). The markers flanking the HaOr7 gene delineated a window of 5,257,546 bp between positions 84,014,359 and $89,271,905$, including the genomic region identified by GWAs. However, a large gap separated the SNPs associated with the lowest p-value, suggestive of local mis-assembly in the current reference genome sequence. We were able to obtain a manually curated genomic sequence of $506 \mathrm{~kb}$, which was not only much smaller than the original $5.25 \mathrm{Mb}$ interval, but it also was more consistent with the location of the markers from the GWA analysis (Supplementary Fig. 1a). In addition, the resistant phenotype in heterozygous plants showed that the allele of HaOr7 conferring resistance was dominant.

We used the corrected genomic sequence to develop new markers in order to define recombination events, which occurred in this interval in a large population of 14,281 F2 plants (Supplementary Fig. 1b) and to reduce the size of the genomic region containing the HaOr7 gene. We genotyped all of the F2 plants, which derived from a cross of susceptible (LSS007) and resistant (LSR007) lines using two markers flanking the HaOr7 gene, and we identified 271 plants showing a recombination event in a $852 \mathrm{~kb}$ genomic sequence that encompassed the $506 \mathrm{~kb}$ target sequence. All 271 recombinant plants were genotyped and each of the recombinant F2 plants was self-crossed to obtain F2:3 families, which were phenotyped in the field (Supplementary Fig. 1c). By comparing the phenotypic and genotypic data, we reduced the window containing the HaOr7 gene to a $114 \mathrm{~kb}$ region between the SF4386 and SF4543 markers. Forty-nine F2 plants showed a recombination event in the $114 \mathrm{~kb}$ target interval. Finally, the HaOr7 gene was located in a genomic region of $55 \mathrm{~kb}$, as determined by two plants carrying a recombination event identified by the flanking markers SF0016 and SF0021 (Fig. 1c). This sequence contained a unique full protein-coding gene (HanXRQChr07g0202981), predicted to encode a protein homolog to AT3G47570 in A. thaliana and Xa21 in rice ${ }^{16}$, encoding a probable leucine-rich repeat receptor-like serine threonine kinase and conferring resistance to Xanthomonas oryzae in rice (the HAOR7 protein showed $44 \%$ of identity and $62 \%$ of similarity with the XA21 protein).

We constructed two Bacterial Artificial Chromosome (BAC) libraries using HaOr7- and HaOr7+ lines, which allowed us to obtain the genomic sequences of $162 \mathrm{~kb}$ for the susceptible parental line and 168 
$\mathrm{kb}$ for the resistant one, both containing the genomic region flanked by markers SF0016 and SF0021. The sequence of the susceptible allele aligned almost perfectly ( $99.71 \%$ identity) along its entire length with the reference genome sequence of the $X R Q$ line, which is susceptible to $O$. cumana race $F$. In contrast, the $168 \mathrm{~kb}$ sequence of the resistance allele aligned poorly to the $162 \mathrm{~kb}$ of the susceptible line and only with coding sequences; no similarity was seen for intergenic regions (Supplementary Fig. 1d). Furthermore, these intergenic regions did not show any similarities with the sequences of other species in the GenBank database. The sequences also showed numerous structural variants. For example, the HanXRQChr07g0202961 and HanXRQChr07g0202971 genes were absent and the HanXRQChr07g0202981 gene was duplicated in the HaOr7+ line. We determined the copy number of the HanXRQChr07g0202981 gene, using qPCR of gDNA on a panel of 79 elite lines whose pedigrees were known for the HaOr7 gene. We found a perfect correlation between the resistance phenotype and duplication of the HanXRQChr07g0202981 gene. These data supported our hypothesis that the HanXRQChr07g0202981 gene could underlie resistance to O. cumana. Moreover, the high differentiation of the genomic sequence of the resistance allele; the intergenic sequences didn't show homology with nucleotidic sequences in databases; and the many structural variants suggest an origin of the resistance allele from a related wild species (Supplementary Fig. 1d).

We then used a panel of wild and cultivated accessions: 132 cultivated Helianthus annuus lines (79 lines whose pedigree for the HaOr7 gene was known, including $43 \mathrm{HaOr} 7+$ lines), 24 wild $\mathrm{H}$. annuus and 14 Helianthus wild relatives. We sequenced $1 \mathrm{~kb}$ in the coding sequence of the three genes HanXRQChr07g0202951, HanXRQChr07g0202811, and HaOr7 on all lines from the panel.

According to phylogenetic (Supplementary Files 1,2,3) and linkage disequilibrium (LD) analyses of the two genes (HanXRQChr07g0202951 and HanXRQChr07g0202811) surrounding HaOr7, only the former was in linkage disequilibrium with the HaOr7 gene (Fig. 1d) and showed a strong decrease in molecular diversity in resistant lines ( $\pi=0$, Supplementary Fig. 2).

The fine mapping and the diversity analysis of the two surrounding genes validate the hypothesis that HanXRQChr07g0202981 gene is responsible for the resistance.

The HAOR7 protein from the resistant sunflower line is annotated as a 1,005 aa protein having LeucineRich Repeat (LRR) domains (IPR013210 and IPR001611), as well as a kinase domain (IPR000719). in silico analyses showed that it contains a transmembrane domain with an extracellular domain in the $\mathrm{N}$-terminal and an intracellular kinase domain in the C-terminal region (Supplementary Fig. 3). All of these results support the hypothesis that the HaOr7 gene encodes a receptor-like membrane protein leading to signal transduction. The susceptible allele of this gene contained a STOP codon leading to a 
truncated protein of 591 aa, which lacked the intracellular kinase domain, such as in the XRQ susceptible line (GenBank accession number XP_021971921), preventing signal transduction.

We sequenced approximately $1 \mathrm{~kb}$ of the HaOr7 gene from either side of the STOP codon in all lines of the panel. We found only three alleles in the HaOr7- lines, all carrying the STOP codon. In contrast, all of the HaOr7+ lines carried the same allele coding a full protein (Supplementary Fig. 3). Compared to HanXRQChr07g0202951, we found less molecular diversity in the HaOr7- lines. Thus, while the proximate origin of the resistance allele in cultivated sunflower is from wild Helianthus, the original origin of the allele remains unknown.

In order to determine which developmental stage was affected by the HaOr7 gene, four stages of development were phenotyped: induction of $O$. cumana seeds germination, root attachment (after 2 weeks of interaction), tubercle development (after 5 weeks of interaction) and emerged shoots (after 14 weeks of interaction). Both parental lines highly induced the germination of broomrape seeds (approx. $98 \%$ for both, Supplementary Fig. 4a). In similar experiments, we counted the number of compatible and incompatible attachments to the sunflower roots. P96 and 2603 sunflower genotypes were added as resistant and susceptible controls, respectively, for this developmental stage. We observed almost entirely compatible attachments in the susceptible line (8.56 per sunflower on average in LSS007, Fig. 2a) compared to the resistant line, which produced almost exclusively incompatible attachments (6.36 per sunflower on average in LSR007). LSS007 showed a susceptibility level similar to the 2603 control genotype (with a mean of 7.23 compatible attachments per sunflower), and LSR007 showed a similar level of resistance as the P96 control genotype (7.35 incompatible attachments per sunflower) (Fig. 2a). Observation of a cross-section of the sunflower root with a broomrape compatible attachment confirmed the connection of $O$. cumana to the vascular system of the root on the susceptible line, while the resistant allele of HaOr7 prevents the connection on the resistant line as shown on an incompatible attachment (Fig. 2b). We further showed that the later stages of the biological cycle of $O$. cumana were affected with respect to tubercle number (Supplementary Fig. 4b) and emerged shoots (Supplementary Fig. 4c) as expected.

In conclusion, we identified the function of the HaOr7 gene as a LRR receptor-like kinase, and we propose a model of the functional mechanism leading to the resistance (Fig. 2c). 


\section{Methods}

\section{Plant material}

Remark: all elite lines (named LSS\#, S\# and LSR\#), developed by Syngenta Seeds, have a known pedigree for the HaOr7 gene. The same resistance allele of the HaOr7 gene was introgressed in all HaOr7+ lines.

\section{Broomrape population}

All phenotyping evaluations in controlled conditions were done with $O$. cumana race $F$ (EcijaF) collected in bulk from several $O$. cumana emerged shoots in a highly infested field from Ecija (Spain, $37.4053000 /-5.1817800)$ in summer 2013.

\section{Segregating populations for mapping and cloning.}

The two inbred lines LSS002 and LSR002, susceptible and resistant to $O$. cumana EcijaF respectively, were crossed to develop a F6 RIL population of 355 plants. We used this population to map the HaOr7 resistance gene on the sunflower genome between the SF3178 and SF3131 SNP markers (Supplementary Table 1).

Seventy-seven F1 plants from the cross between the 2 inbred lines LSS007 and LSR007, susceptible and resistant to $O$. cumana EcijaF respectively, were selfed and we genotyped their corresponding 16,056 F2 plants, with SF3178 and SF3946 SNP markers (close to the SF3131 marker). We discarded 1,775 F2 plants that did not segregate as expected; they had been obtained from 9 selfed F1, originating from failed crosses. The remaining 14,281 F2 plants showed expected allele segregation ratios for both markers. We identified 290 F2 plants with a recombination event between the SF3178 and SF3946 markers (Fig. 1b) but only 271 produced enough seeds after selfing. After fine-mapping of the HaOr7 gene, we selected 49 F2:3 families that showed a recombination event in a restricted genomic region. For each of them, we selected two F3:4 families, homozygous for both alleles of the closest segregating markers. Finally, we kept two F3:4 families that enabled the cloning of the HaOr7 genomic region (Supplementary Table 2).

Diversity panel for GWAs and phylogenetic analysis

For the mapping of the HaOr7 gene, we used a set of 55 inbred lines given by Syngenta Seeds, 34 were resistant due to the HaOr7 gene and 21 were susceptible to 0 . cumana EcijaF.

For the phylogenetic analysis of HaOr7 gene and its genomic region, we used a set of 170 Helianthus accessions. The 170 accessions were composed of 132 cultivated Helianthus annuus lines (79 lines 
whose pedigree for the HaOr7 gene was known, including $43 \mathrm{HaOr} 7+$ lines), 24 wild $\mathrm{H}$. annuus and 14 Helianthus wild relatives (Supplementary Table 3).

\section{Phenotyping for broomrape resistance}

\section{Phenotyping in the field}

We evaluated the plants in the same highly infested field in Ecija (Spain, 37.4053000 / -5.1817800 ) between 2015 and 2017. In order to validate the level of the $O$. cumana natural inoculum and to identify any spatial variations of the natural inoculum in the soil, we used resistant and susceptible controls. We grew a row of susceptible control plants every 12 rows and we always found infected plants in these rows.

In spring 2015 , we phenotyped the F6 RIL population used for the mapping, 18 plants in average, classified as uninfected or infected (Supplementary Table 2). We phenotyped the recombinant families in spring 2016, of at least 20 F3 plants per family with 2 replicates when possible (Supplementary Table 4). After approximately four months of growth, we phenotyped each sunflower plant. The plant was noted as susceptible if five or more $O$. cumana plants emerged. For sunflower plants with less than four emerged shoots, we precisely counted the number of emerged shoots. Sunflower with less than three emerged shoots was considered as resistant. An intermediate phenotype consisted of three or four $O$. cumana emerged shoots per sunflower plant. The same protocol was used to confirm the phenotype of the selected F3:4 families on 2 replicates of at least 20 plants during spring 2017 (Supplementary Table 5).

We phenotyped all cultivated public $H$. annuus accessions from the diversity panel, which has been used for diversity and phylogenetic analyses, with 10 plants per accessions. The number of $O$. cumana emerged shoots per sunflower was precisely counted after about 4 months of growth.

Preconditioning of $O$. cumana seeds for phenotyping sunflowers in controlled conditions

O. cumana seeds were placed during 7 days at $22{ }^{\circ} \mathrm{C}$ in the dark, in a moistened atmosphere. For Minirhizotron experiments ${ }^{7}$, we surface-sterilized the seeds with bleach at 0.7 chlorometric degree during 5 minutes, before rinsing them 2-3 times with sterile water.

Phenotyping in controlled conditions

Mini-rhizotron experiments were used to phenotype the early stages of the interaction for the parental lines LSS007 and LSR007. We placed the root system of 7-days old sunflower plantlets grown in a mix 
of sand and vermiculite on a moistened filter paper with $10 \mathrm{mg}$ of preconditioned $O$. cumana seeds. Sunflower plants were grown for 2 more weeks at a constant temperature of $22{ }^{\circ} \mathrm{C}$, with a day/night cycle of $16 \mathrm{~h} / 8 \mathrm{~h}$ and watered with a $0.5 \mathrm{X}$ Long-Ashton Nutrient Solution (LANS) ${ }^{17}$. For germination assays, Mini-rhizotrons of LSR007 and LSS007 were placed in different boxes to avoid contamination of the root exudates between genotypes. We counted, for about $100 \mathrm{O}$. cumana seeds, the number of germinated and non-germinated seeds on the filter under a binocular microscope (Leica LED2000). For the attachment stage, we added P-96 and 2603 lines as resistant and susceptible controls respectively. We then counted the number of $O$. cumana attachments on the sunflower root under a binocular microscope (Leica LED2000).

\section{Microscopic analyses}

Root fragments of inoculated sunflower in mini-rhizotrons, containing incompatible attachments from the LSR007 line or compatible attachments from the LSS007 line were sampled 14 days after inoculation. Samples were fixed in FAA (10 \% formaldehyde, $5 \%$ acetic acid, $50 \%$ ethanol), dehydrated in an alcohol series and embedded in Technovit 7100 (Heraeus Kulzer, Hanau, Germany) according to the manufacturer's instructions. Sections $(10 \mu \mathrm{m})$ were stained with $0.2 \%$ toluidin blue for 3 minutes and rinsed thoroughly. Dried sections were mounted in DPX mountant for histology (ref 4481, Fluka, France) and observed with a Zeiss Axioplan 2 light microscope (Carl Zeiss, Le Pecq, France).

\section{Sequence analysis}

All genes IDs and genomic locations refer to the reference sunflower genome sequence (https://www.heliagene.org/HanXRQ-SUNRISE/) ${ }^{15}$.

\section{Genomic sequence assembly}

Using the sequences of the markers that surrounded the HaOr7 gene after mapping (Fig. 1b), we identified two genomic contigs from the assembly of PacBio sequencing data of the XRQ sunflower line reference genome ${ }^{15}$, by BLAST alignments ${ }^{22}$. We aligned by BLAST the sequence extremities of these contigs using different assemblies obtained at different depths of PacBio sequencing. Finally, we assembled the contig sequences using Minimus software (AMOS software package) as a single sequence of 852,580 nucleotides that fully covered the genomic region containing $\mathrm{HaOr} 7$ between the two SNP markers (HaOr7 contig). 
For structural annotation, genes were automatically annotated using Eugene (release 4.2) software using the same protocol as the one described in Badouin et al. (2017) ${ }^{15}$. Protein coding genes were functionally annotated with InterProScan (release 63) to predict protein domains, including transmembrane domains and signal peptides.

\section{Statistical and Diversity analysis}

For physiological tests, variances of all groups have been compared with F-test $(p<0.05)$, and their means with t-test for unpaired individuals. To compare means of compatible and incompatible attachements within genotypes, we used t-test for paired individuals.

We performed association mapping using Plink 1.07 software (options --assoc -adjust) which generated a file of adjusted $p$-values corrected by a Bonferroni test (Supplementary Table 7).

Linkage disequilibrium was calculated on 22 lines (13 HaOr7+ lines and 9 HaOr7- lines) between SNPs found in the sequences of the 3 genes HanXRQChr07g0202811, HaOr7 and HanXRQChrO7g0202951 sequences (Supplementary Table 8). The significance threshold of linkage disequilibrium was obtained by randomly sampling 10,000 pairs of independent SNPs between one located in one of the 3 genes on chromosome 7 and one located in the HanXRQChr17g0540231 gene on chromosome 17, calculating the squared Pearson's correlation $r^{2}$ between each pair, and taking the $99.9 \%$ quantile of the 10,000 distribution as the threshold. We obtained a threshold of 0.19 .

Data availability statement:

All data generated or analysed during this study are included in this published article (and its supplementary information files). 


\section{References:}

1. Subrahmaniam, H. J. et al. Plant J. 12, 3218-3221 (2017).

2. Westwood, J. H., Yoder, J. I., Timko, M. P. \& dePamphilis, C. W. Trends Plant Sci. 15, 227-235 (2010).

3. Yoshida, S., Cui, S., Ichihashi, Y. \& Shirasu, K. Annu. Rev. Plant Biol. 67, 643-667 (2016).

4. Parker, C. Pest Manag. Sci. 65, 453-459 (2009).

5. Li, J. \& Timko, M. P. Science (80-. ). 325, 1094 (2009).

6. Rispail, N. et al. New Phytol. 173, 703-712 (2007).

7. Louarn, J. et al. Front. Plant Sci. 7, 1-14 (2016).

8. Pérez-Vich, B. et al. Theor. Appl. Genet. 109, 92-102 (2004).

9. Fernández-Martínez, J. M., Domínguez, J., Pérez-Vich, B. \& Velasco, L. Helia 33, 1-12 (2008).

10. Tang, S. et al. Crop Sci. 43, 1021-1028 (2003).

11. Rodríguez-Ojeda, M. I. et al. Weed Res. 53, 322-327 (2013).

12. Flor, H. H. North 9, 275-296 (1971).

13. Martín-Sanz, A., Malek, J., Fernández-Martínez, J. M., Pérez-Vich, B. \& Velasco, L. Front. Plant Sci. 7, 1-9 (2016).

14. Molinero-Ruiz, L. et al. Spanish J. Agric. Res. 13, 1-19 (2015).

15. Badouin, H. et al. Nature 546, 148-152 (2017).

16. Song, W. et al. Science (80-. ). 270, 2-4 (1995).

17. Hewitt, E. J. Commonw. Bur. Hortic. 22, 547 (1966).

18. Peterson, D. G., Tomkins, J. P., Frisch, D. A., Wing, R. A. \& Paterson, A. H. J. Agric. Genomics 5, (2000).

19. Schweiger, W. et al. Theor. Appl. Genet. 129, 1607-1623 (2016).

20. Chalhoub, B., Belcram, H. \& Caboche, M. Plant Biotechnol. J. 2, 181-188 (2004).

21. Gonthier, L. et al. BMC Res. Notes 225, (2010).

22. Altschul, S. F., Gish, W., Miller, W., Myers, E. w. \& Lipman, D. J. J. Mol. Biol. 215, 403-410 (1990).

23. Chin, C. S. et al. Nat. Methods 10, 563-569 (2013).

24. You, F. M. et al. BMC Bioinformatics 9, 1-13 (2008). 


\section{Acknowledgement}

We thank all the following colleagues from LIPM (INRA, France) for their help: Erika Sallet for her help in genes annotation, Jérôme Gouzy for his advices in sequence analysis and his critical review of the manuscript, Nicolas Pouilly for his advices in genotyping experiments, Nemo Peeters (director of the Toulouse Plant Microbe Phenotyping platform), Fabrice Devoilles and Mehdi Khafif for their help for some phenotyping experiments in greenhouse. We thank Loren Rieseberg (UBC, Canada) for his critical review of the manuscript and for improving the writing. This work was supported by INRA, Syngenta seeds and the French Laboratory of Excellence project "TULIP" (ANR-10-LABX-41; ANR-11-IDEX-0002$02)$. 


\section{Author contributions:}

P.D. contributed to statistical analysis, to genotyping, sequencing and phenotyping experiments. S.V. developed BAC libraries and performed their screening. C.C. performed the sequencing of the BAC clones. S.Cauet assembled the sequences of the BAC clones. S.Carrère annotated genes from the genomic sequences. J.B. contributed to phylogenetic analysis. M.C.B. and F.G. contributed to sunflower plants crossing and growing. J.C.R. contributed to the genotyping experiments. M.L.S. contributed to field phenotyping experiments. M.C. and M.C.A. performed microscopic experiments. P.P.E. calculated linkage disequilibrium. C.P. contributed to the supervising of the map-based cloning analysis. B.P.V. and L.V. contributed to the phenotyping for phylogenetic analysis. H.B. supervised the genomic analysis on BAC clones. J.P. and S.M. designed all experiments and managed the project. All authors contributed to the writing of the manuscript.

\section{Competing interests:}

The authors declare no competing interests. 


\section{Figure legends:}

\section{Figure 1: Identification of the HaOr7 gene onto the sunflower genome}

\section{a, Association mapping of the HaOr7 gene.}

A panel of 55 sunflower lines (21 HaOr7- lines and 34 HaOr7+ lines) were genotyped with an AXIOM ${ }^{\circledR}$ array of 586,985 SNPs. A total of 305,201 SNPs were polymorphic on the whole panel and were located with a single position on the 17 chromosomes of the reference genome sequence. The $p$-value $(p)$ of the association test was calculated and adjusted using the Bonferroni correctiontest (Supplementary Table 7). The blue line corresponds to a threshold of $10^{-5}$ for $-\log _{10}(p)$ and the red line to a threshold of $10^{-7}$. In total, 82 SNPs were significantly associated with the resistance. All of them were located on chromosome 7 in a 1,122,175 bp window containing 363 SNPs from the $\mathrm{AXIOM}^{\circledR}$ array.

\section{b, Mapping of the HaOr7 gene in a segregating biparental population.}

A population of 355 F6 Recombinant Inbred Lines was genotyped with 40 SNP markers located on chromosome 7. The HaOr7 gene was mapped in a $2.9 \mathrm{cM}$ interval, located in the same genomic region of chromosome 7 as the one mapped by GWAs.

\section{c, Fine-mapping of the HaOr7 gene in a large $\mathrm{F} 2$ population.}

The genotype and phenotype of the two recombinant plants 48657 and 61961 allowed the mapping of the HaOr7 gene in a $55 \mathrm{~kb}$ window between the SF0016 and the SF0021 markers, containing one candidate gene on the resistant sequence: HanXRQChr07g0202981. Gene positions, from CDS alignments, are represented by gray boxes, and the transcription strand is shown with arrows. The resistant allele $(R)$ is indicated in orange, and the susceptible allele $(S)$ is shown in blue.

\section{d, Linkage Disequilibrium (LD) analysis}

Heatmap of $r^{2}$ values between all possible pairs of SNPs in the three candidate gene sequences greater than a significance threshold of 0.19 ( $p$-value $<0.001)$.

Figure 2: HaOr7 acts by preventing connection of $O$. cumana to the root vascular system.

\section{a, Evaluation of attachments of $O$. cumana to the sunflower roots.}

In mini-rhizotron systems, we counted the number of incompatible attachments (IA, brown) and compatible attachments (CA, pale yellow) on $n$ replicates. We used the 2603 and P96 lines as 
susceptible and resistant controls, respectively. Vertical lines and/or dots show minima and maxima values, box lines show the first and third quartiles and the middle line shows the median value. Significant differences with the susceptible parental line (LSS007) are indicated by $* * *: p<0.01, * *$ : $\mathrm{p}<0.05$, calculated with Welch's two-sided t-test.

\section{b, Microscopic sunflower root cross-sections of compatible and incompatible attachments.}

Compatible and incompatible attachments were obtained from the susceptible (LSS007) and resistant (LSR007) lines respectively in mini-rhizotron experiments. Three compatible attachments and 11 incompatible attachments were sampled.

VS: Vascular system of the sunflower root. Oc: Orobanche cumana. The arrow indicates the connection of $O$. cumana to the VS in the susceptible line. Bar $=100 \mu \mathrm{m}$.

c, Molecular model leading to resistance by preventing the connection of $O$. cumana to the vascular system in sunflower roots

An avirulence protein (AVROR7) from $O$. cumana is recognized by the LRR domain of the HAOR7 protein enabling the signal transduction through its kinase domain and leading to resistance. The truncated protein in susceptible sunflowers lacks the transmembrane and the kinase domains. The signal transduction is not allowed, leading to the connection of $O$. cumana to the vascular system in sunflower roots. 